\title{
Video-Assisted Thoracic Surgery as a Less-Invasive Management for Acute Hemothorax in Blunt Trauma
}

\author{
Yoshihiko Kurimoto ${ }^{1}$, Atsushi Watanabe ${ }^{2}$, Tetsuya Koyanagi ${ }^{2}$, Toshiro Ito $^{2}$, Tetsuya Higami ${ }^{2}$, \\ Kunihiko Maekawa ${ }^{1}$, Katsutoshi Tanno ${ }^{1}$, Yasufumi Asai ${ }^{1}$ \\ ${ }^{1}$ Department of Traumatology and Critical Care Medicine, Sapporo Medical University, Sapporo, Japan \\ ${ }^{2}$ Department of Thoracic and Cardiovascular Surgery, Sapporo Medical University, Sapporo, Japan \\ Email: kurimoto@sapmed.ac.jp
}

Received January 4, 2012; revised January 18, 2012; accepted January 28, 2012

\begin{abstract}
Purpose: We report our experience of Video-assisted thoracic surgery (VATS) to treat acute blunt traumatic hemothorax using mini-thoracotomy. Methods: We designed a prospective study to determine if VATS with mini-thoracotomy benefits for patients with blunt traumatic hemothorax compared with conventional repair through full thoracotomy. Twenty-five patients underwent emergency operation for acute hemothorax from 2000. Five patients with less than 5\% probability of survival (PS) were excluded, leaving 20 as the subjects. Results: Ten patients underwent conventional thoracotomy (conventional group) and the other 10 patients underwent VATS with mini-thoracotomy (VATS group). There was no difference between conventional group and VATS group in injury severity score (29.1 and 27.0) or PS (81.2\% and $80.7 \%$ ). Hospital mortality rates were $10 \%$ in conventional group and $0 \%$ in VATS group (N.S). Total amounts of intra-operative bleeding and post-operative transfusion until day 7 were $735 \mathrm{ml}$ and 19.3 units in conventional group and $303 \mathrm{ml}$ and 9.2 units in VATS group respectively (N.S). The length of ICU stay was 9.7 days in conventional group and 5.9 days in VATS group (N.S). Conclusion: VATS with mini-thoracotomy can be alternative for patients with blunt traumatic hemothorax in most emergency operations.
\end{abstract}

Keywords: Hemothorax; Trauma; Video-Assisted Thoracic Surgery; Emergency Surgery; Critical Care

\section{Introduction}

Thoracoscopy has long been recognized as a useful tool for diagnosis of penetrating diaphragmatic injury [1]. Recent reviews show that video-assisted thoracic surgery (VATS) affords diagnostic and therapeutic benefits when used in the subacute management of trauma patients. It is believed that VATS can be used to diagnose diaphragmatic injury and also treat pleural space complications, early clotted hemothorax, persistent pneumothorax and ongoing bleeding $[2,3]$. However, VATS has been employed for only a limited category of trauma patients namely hemodynamically stable and non-emergency cases. Given that VATS is technically demanding and requires a long time to achieve temporary hemostasis in the pleural cavity in an emergency situation with trauma patients, the limitations on its use mentioned above would seem to be rational.

In our department, it has been the policy that VATS can be applied even for an emergency operation with a mini-thoracotomy. In particular, blunt trauma patients who often suffer from other associated injuries should to the greatest extent possible be managed by a minimally invasive approach. In this study, we introduce our minimally invasive approach in emergency operations for hemothorax caused by blunt trauma.

\section{Patients and Methods}

We designed a prospective clinical study to determine if VATS with mini-thoracotomy is more beneficial than conventional repair with a full thoracotomy for patients with blunt traumatic hemothorax requiring an emergency operation. Selection of operative procedures was dependent on the availability of a VATS system on the day of the emergency operation. Although our selection priority of initial management is VATS with a mini-thoracotomy, the VATS system is not commonly available for emergency surgery in our facility. For example, when scheduled VATS for another elective patient is on going or when a VATS system is under sterilization, the VATS system cannot be employed for emergency patients. The level of entry for mini-thoracotomy is decided on the basis of suspected intra-thoracic injury and the length of incision, approximately $5 \mathrm{~cm}$ to $10 \mathrm{~cm}$, is decided on the basis of each patient's body fat content. Emergency he- 
mostasis under VATS is carried out through both thoraco-ports and an intercostal space opened by a minithoratotomy. Mini-thoracotomy is necessary to provide a surgical field view through thoracoscopy quickly and effectively following initial evacuation of blood in a pleural cavity. In cases of multiple organ injuries, surgical repairs are performed simultaneously as much as possible although priority is given to critical injuries. To facilitate emergency surgery for multiple injuries, the patients are generally placed in the supine position or the semi-lateral position although this is not appropriate for VATS (Figure 1).

Since January 2000, 101 patients with blunt traumatic hemothorax excluding cardiac and aortic injuries have been admitted to our emergency medical center in the university hospital and initially managed by tube thoracotomy. Our emergency medical center accepts only patients who are determined by emergency medical technicians or referring physicians to be seriously sick or injured, and are transferred by ambulance or helicopter. Walk-in patients are not eligible for admittance to our department. 25 (24.8\%) of the admitted patients underwent emergency operations for acute hemothorax. Five patients with less than a $5 \%$ probability of survival (PS), which was calculated by the TRISS method [4], were excluded, and the remaining 20 were the subjects of this study. To compare conventional thoracotomy with VATS with mini-thoracotomy as an initial treatment, early mortality, operating time (limited only to the procedure for hemothorax), total amount of transfusion (including both MAP and frozen fresh plasma) and length of ICU stay were analyzed statistically.

The data are presented as means plus range or \pm standard deviations. The Wilcoxon test or the chi-squared test was used to compare data in the two groups using JMP5.1 statistical software (SAS Institute Inc, Cary, NC).

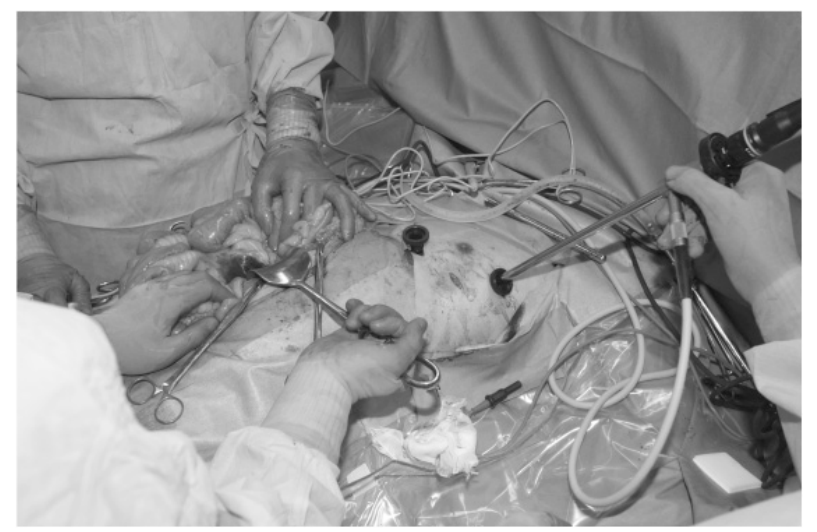

Figure 1. Operating picture of video-assisted thoracic surgery for multiple injuries. To perform an emergency concomitant surgery, a patient is generally placed in the supine position or semi-lateral position only of upper body.

\section{Results}

Ten patients underwent conventional thoracotomy (the conventional group) and the other 10 patients underwent VATS with mini-thoracotomy (the VATS group). There was no significant difference between the conventional group and the VATS group in mean age (50.7 years and 49.9 years), gender (8 male and 6 male), mean injury severity score (ISS) (29.1 and 27.0), revised trauma score (RTS)(7.05 and 7.01), or mean PS (81.2\% and 80.7\%), respectively (Table 1).

One patient from the conventional group died on the 18th day after surgery. The patient was a 20-year-old female pedestrian hit by a car. Despite associated traumatic subarachnoid hemorrhage, her preoperative hemodynamic state was relatively stable (RTS; 7.55, PS; 94.3\%) in the emergency room. Sudden hemoptysis occurred just before the surgery, which caused hypoxia. Extra-corporeal membrane oxygenation (ECMO) was employed to combat against critical hypoxia after emergency surgery. Although ECMO was weaned at the 11th day after operation, the patient died due to MOF. Overall hospital mortality rates were $10 \%$ in the conventional group and $0 \%$ in the VATS group (N.S).

There were eight patients, four from each group, who underwent surgical or trans-catheter interventions for other associated injuries simultaneously or on the same day of emergency surgery for hemothorax (Table 2).

Hemothorax-related operating time was $143.0 \pm 65.3$ minutes in the conventional group and $108.5 \pm 36.4$ minutes in the VATS group (N.S). Total amounts of operative bleeding and post-operative transfusion until day 7 were $735 \pm 693 \mathrm{ml}$ and $19.3 \pm 17.7$ units, respectively, in the conventional group and $303 \pm 511 \mathrm{ml}$ and $9.2 \pm 7.6$ units in the VATS group (N.S). The mean length of ICU stay was $9.7 \pm 11.6$ days in the conventional group and $5.9 \pm 5.9$ days in the VATS group (N.S) (Figure 2).

There were four patients who had to stay in ICU more than 14 days, three (30.0\%) from the conventional group and one (10.0\%) from the VATS group (N.S). Ventilator-associated pneumonia (VAP) occurred in one patient in the conventional group (10.0\%), who was a 75 -yearold female and suffered from a thoracic vertebral fracture. The patient remained in ICU for 39 days after the operation.

\section{Discussion}

In the present study, we have reported on the superiority of VATS with a mini-thoracotomy over conventional thoracotomy as an emergency treatment for traumatic hemothorax. VATS may be beneficial especially for multiple trauma patients in terms of its feasibility for simultaneous surgery for associated injuries and favorable postoperative course. Among 20 patients, one early 
Table 1. Patients' characteristics.

\begin{tabular}{|c|c|c|c|c|c|c|c|c|c|}
\hline Conventional & & & Cause of & Preoperative & Time from & Cause of & & & \\
\hline group & Age & Gender & injury & intubation & accident (hours) & hemothorax & ISS & RTS & PS \\
\hline 1 & 47 & Male & Automobile & $(-)$ & 3 & Diaphragma & $29(\mathrm{C} 4, \mathrm{~A} 3, \mathrm{E} 2)$ & 7.84 & 94.7 \\
\hline 2 & 50 & Male & Automobile & $(-)$ & 20 & Lung & 18 (F1,C4,E1) & 6.37 & 96.1 \\
\hline 3 & 20 & Female & Hit by car & $(-)$ & 24 & Lung & 34 (H3,C5) & 7.55 & 94.3 \\
\hline 4 & 55 & Male & Bicycle & $(-)$ & 12 & Chest wall & 9 (C3) & 7.84 & 96.8 \\
\hline 5 & 77 & Male & Fall & $(-)$ & 12 & Lung & $29(\mathrm{C} 4, \mathrm{~A} 3, \mathrm{E} 2)$ & 7.84 & 84.9 \\
\hline 6 & 30 & Male & Automobile & $(+)$ & 20 & Diaphragma & 34 (C5,A3) & 6.61 & 86.9 \\
\hline 7 & 58 & Male & Automobile & $(+)$ & 4 & Lung & 34 (C5,E3) & 6.82 & 69.3 \\
\hline 8 & 27 & Male & Fall & $(+)$ & 3 & Chest wall & $41(\mathrm{C} 4, \mathrm{~A} 3, \mathrm{E} 4)$ & 7.84 & 92.2 \\
\hline 9 & 75 & Female & Fall & $(+)$ & 4 & Vertebra & 25 (C4,A3) & 6.09 & 65.5 \\
\hline 10 & 68 & Male & Fall & $(-)$ & 12 & Lung & 38 (H2,C5,E3) & 5.68 & 31.6 \\
\hline mean \pm S.D. & $50.7 \pm 20.0$ & & & & $11.4 \pm 7.9$ & & $29.1 \pm 9.6$ & $7.05 \pm 0.83$ & $81.2 \pm 20.7$ \\
\hline \multicolumn{10}{|l|}{ VATS group } \\
\hline 11 & 60 & Male & Automobile & $(+)$ & 3 & Diaphragma & 14 (C3,A2,E1) & 7.84 & 95.2 \\
\hline 12 & 52 & Female & Automobile & $(-)$ & 24 & Diaphragma & 26 (F1,C4,A3) & 7.55 & 97.0 \\
\hline 13 & 67 & Female & Fall & $(-)$ & 3 & Lung & 19 (H3,C3,E1) & 7.55 & 91.1 \\
\hline 14 & 48 & Male & Motorcycle & $(+)$ & 2 & Lung & $29(\mathrm{C} 4, \mathrm{~A} 2, \mathrm{E} 3)$ & 6.08 & 88.6 \\
\hline 15 & 35 & Male & Motorcycle & $(-)$ & 9 & Lung & 25 (C4,E3) & 7.84 & 97.8 \\
\hline 16 & 71 & Male & Automobile & $(-)$ & 3 & Lung & $24(\mathrm{C} 4, \mathrm{~A} 2, \mathrm{E} 2)$ & 6.61 & 75.9 \\
\hline 17 & 55 & Female & Fall & $(+)$ & 24 & Diaphragma & 54 (H5,C5,E2) & 5.03 & 6.7 \\
\hline 18 & 18 & Male & Motorcycle & $(-)$ & 12 & Lung & 17 (C4,A1) & 7.55 & 99.1 \\
\hline 19 & 30 & Female & Automobile & $(-)$ & 7 & Lung & 26 (F1,C4,E3) & 7.11 & 95.8 \\
\hline 20 & 63 & Male & Bicycle & $(-)$ & 20 & Lung & 36 (H4,F2,C4) & 6.90 & 59.5 \\
\hline mean \pm S.D. & $49.9 \pm 17.3$ & & & & $10.7 \pm 8.9$ & & $27.0 \pm 11.4$ & $7.01 \pm 0.90$ & $80.7 \pm 28.8$ \\
\hline
\end{tabular}

ISS: Injury Sevirity Score, RTS: Revised Trauma Score, PS: Probability of Survival.

Table 2. Additional intervention or concomittant surgery for associated injury.

\begin{tabular}{cc}
\hline Case $^{*}$ & Other intervention or surgery on the same day \\
\hline 1 & Splenectomy, Left nephrectomy \\
7 & External fixation of both-leg fracture \\
8 & Trans-catheter arterial embolization for pelvic fracture \\
10 & Definite fixation of lower leg open fracture \\
11 & Repair of liver and mesenteric injury \\
14 & External fixation of pelvic fracture \\
17 & Thoracic endovascular aortic repair for aortic injury \\
19 & External fixation of leg fracture \\
\hline
\end{tabular}

Asterisk ${ }^{*}$ means that a number of case reflects the number on Table 1. death was experienced only in the conventional group. The patient was a 20 -year-old female in relatively stable condition despite massive right hemothorax in the emergency room. We usually manage trauma patients with hemoptysis using a bronchial blocker or separated intubation to prevent hypoxia secondary to hemoptysis affecting the non-injured side of the lung. However, sudden preoperative deterioration in the operating room made it necessary to start right-sided thoracotomy without separated intubation. We reviewed the patient's clinical course and concluded that standard thoracotomy was not a cause of early death in this case. As such, in the present study we have not attempted to demonstrate that VATS improves the early mortality rate for traumatic hemothorax when compared with conventional thoracotomy. 

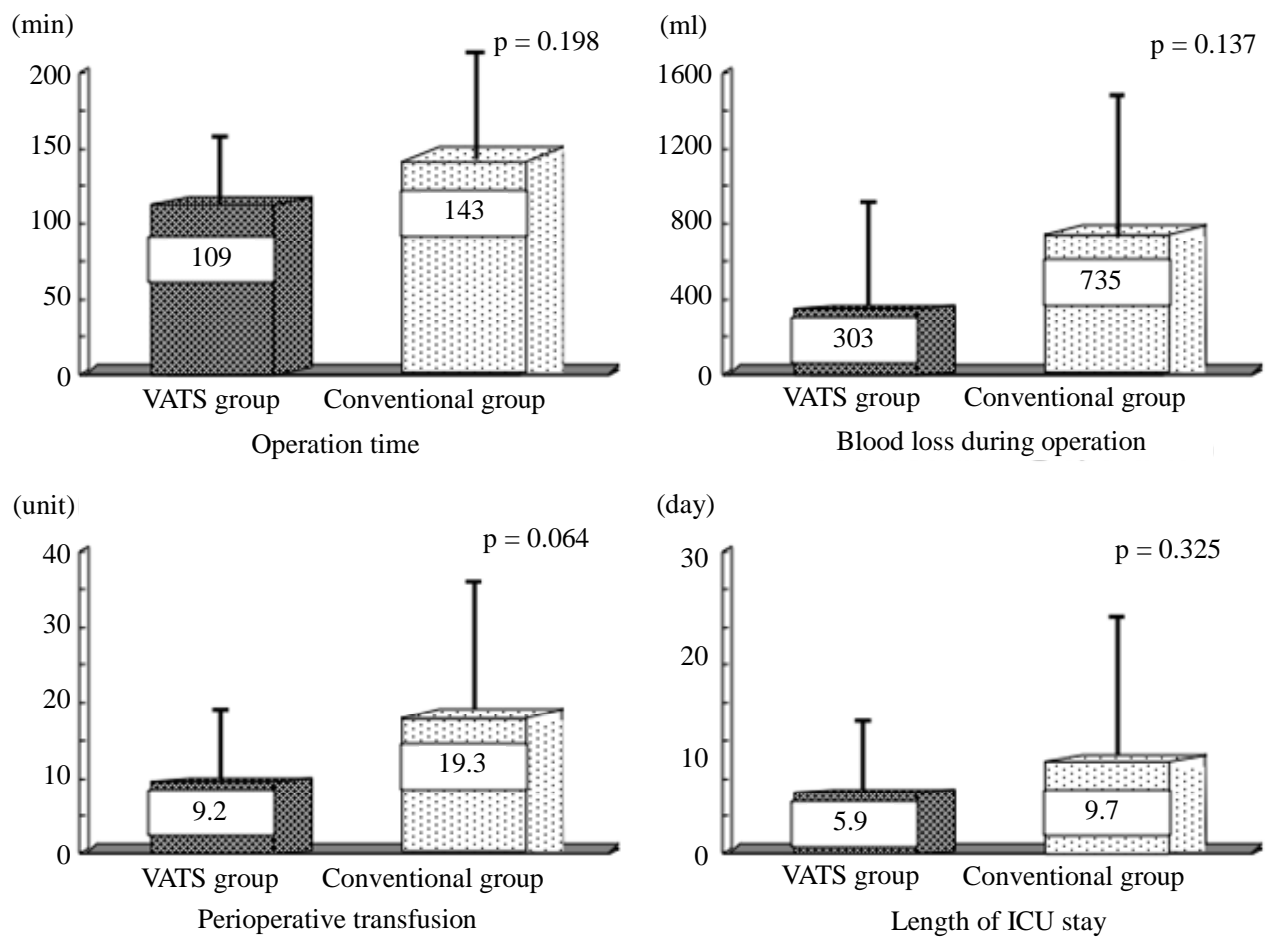

Figure 2. Comparison of perioperative variables. There was no variable suggesting that video-assisted thoracic surgery with mini-thoracotomy in emergency surgery would be more harmful than the conventional approach.

Generally, chest tube thoracotomy can be an initial management for $80 \%$ - $85 \%$ of all chest-injured patients [5]. However, on-going bleeding, retained clotted hemothorax, persistent pneumothorax, and empyema will necessitate thoracotomy in $20 \%$ - $30 \%$ of these initially conservatively treated patients [6]. Improved recent surgical results in the elective operation has led us to choose less-invasive procedures not only in non-trauma surgeries but even for trauma surgeries as well. Due to recent advancements in VATS, most chest trauma patients are candidates for VATS in both elective and urgent operations $[7,8]$. The rate of conversion to thoracotomy is reportedly around $20 \%[7,9,10]$ although one report exists of penetrating diaphragmatic injury being diagnosed and repaired $100 \%$ by VATS [11]. However, emergency cases are fundamentally different. A conversion from VATS to thoracotomy means an increased amount of blood loss and longer operation time. If the conversion rate is up as high as $10 \%$, VATS cannot be validated as a treatment of choice for emergency hemothorax.

Diaphragmatic injury, which was the main cause of hemothorax in $25 \%$ of the cases in our study, is a unique injury because of the many differences in diagnosis and treatment between blunt and penetrating trauma. Blunt left diaphragmatic injury can be detected easily by simple chest X-ray film. On the other hand, it is often difficult to detect penetrating diaphragmatic injury and that is why thoracoscopy or laparoscopy has been employed for patients with penetrating thoraco-abdominal injuries. Although there are some reports in which the usefulness of thoracoscopy or laparoscopy is described [12,13], laparotomy is often necessary even for patients with penetrating injuries [14], and much more often for patients with blunt diaphragmatic injuries in order to repair the associated abdominal organ injury and to restore abdominal organs stacked in the left pleural cavity. To reduce surgical invasiveness in patients with blunt diaphragmatic injuries without delay of temporary hemostatsis, a combination of laparotomy and VATS with or without a mini-thoracotomy may be an alternative to standard thoracotomy.

There are two considerable problems when VATS is applied for blunt traumatic hemothorax, especially in emergency operations, such as in the present study. Immediate temporary hemostasis is crucial and these blunt trauma patients are generally placed on the operating table in the supine position because of multiple trauma or the possibility of aggravating abdominal injuries. We experienced one patient who died of liver injury, for which there was no indication of surgical repair preoperatively, during open repair of the lacerated lung in the lateral position (data not shown). Since then, we have attempted emergency VATS with a mini-thoracotomy in the supine position for multiple traumatic patients. The supine position allows for the simultaneously repair of multiple injuries in order to reduce operating time and blood loss. Con- 
trary to a study of spontaneous hemopneumothorax, when compared with a VATS alone procedure, VATS with a mini-thoracotomy was reported to reduce operation time without prolonging either periods of chest tube placement or length of hospital stay [15]. Although the present study excluded critical patients with a survival rate probability of less than $10 \%$, there was no increase in the amount of blood transfused during the perioperative periods in the VATS group compared with the conventional thoracotomy group. Moreover, there was a tendency toward shorter ICU stays among patients in the VATS group.

There are several limitations in this study. For example, due to it being a single center study, the number of the patients was very small. There were no statistically significant differences between the groups. We suspect that multiple trauma causes the difficulties in analyzing the results of this study. Moreover, although this is a prospective study, it is not randomized. However, we propose that VATS with a mini-thoracotomy is a less-invasive alternative treatment for emergency traumatic hemothorax because, at least, there was few disadvantage when compared with conventional thoracotomy.

In conclusion, VATS with mini-thoracotomy should be considered a viable option as a less-invasive treatment for emergency traumatic hemothorax.

\section{REFERENCES}

[1] M. G. Ochsner, G. S. Rozycki, F. Lucente, D. C. Wherry and H. R. Champion, "Prospective Evaluation of Thoracoscopy for Diagnosing Diaphragmatic Injury in Thoracoabdominal Trauma: A Preliminary Report,” Journal of Trauma, Vol. 34, No. 5, 1993, pp. 704-709. doi:10.1097/00005373-199305000-00013

[2] N. Ahmed and D. Jones, "Video-Assisted Thoracic Surgery: State of the Art in Trauma Care,” Injury, Vol. 35, No. 5, 2004, pp. 479-489. doi:10.1016/S0020-1383(03)00289-4

[3] A. V. Manlulu, T. W. Lee, K. H. Thung, R. Wong and A. P. Yim, "Current Indications and Results of VATS in the Evaluation and Management of Hemodynamically Stable Thoracic Injuries,” European Journal of Cardio-Thoracic Surgery, Vol. 25, No. 6, 2004, pp. 1048-1053. doi:10.1016/j.ejcts.2004.02.017

[4] C. R. Boyd, M. A. Tolson and W. S. Copes, "Evaluating Trauma Care: The TRISS Method. Trauma Score and the Injury Severity Score,” Journal of Trauma, Vol. 27, No. 4, 1987, pp. 370-378. doi:10.1097/00005373-198704000-00005

[5] K. L. Mattox, M. J. Wall and L. R. Pickard, “Thoracic
Trauma,” In: D. Feliciano, E. Moore and K. L. Mattox, Eds., Trauma, 3rd Edition, Appleton \& Lang, Stanford, 1996, pp. 345-354.

[6] E. H. Carrillo, B. T. Heniford, S. W. Etoch, H. C. Polk Jr., D. L. Miller, F. B. Miller and J. D. Richardson, "VideoAssisted Thoracic Surgery in Trauma Patients," Journal of American College of Surgery, Vol. 184, No. 3, 1997, pp. 316-324.

[7] E. H. Carrillo and J. D. Richardson, "Thoracoscopy for the Acutely Injured Patient,” American Journal of Surgery, Vol. 190, No. 2, 2005, pp. 234-238. doi:10.1016/j.amjsurg.2005.05.018

[8] L. Lang-Lazdunski, J. Mouroux, F. Pons, G. Grosdidier, E. Martinod, D. Elkaïm, J. Azorin and R. Jancovici, "Role of Videothoracoscopy in Chest Trauma," Annals of Thoracic Surgery, Vol. 63, No. 2, 1997, pp. 327-333. doi:10.1016/S0003-4975(96)00960-5

[9] J. A. Kern, C. G. Tribble, W. D. Spotnitz, B. M. Rodgers and T. M. Daniel, "Thoracoscopy in the Subacute Management of Patients with Thoracoabdominal Trauma," Chest, Vol. 104, No. 3, 1993, pp. 942-945. doi:10.1378/chest.104.3.942

[10] P. H. Navsaria, R. J. Vogel and A. J. Nicol, “Thoracoscopic Evaluation of Retained Posttraumatic Hemothorax,” Annals of Thoracic Surgery, Vol. 78, No. 1, 2004, pp. 282-286. doi:10.1016/j.athoracsur.2003.11.029

[11] M. Martinez, J. E. Briz and E. H. Carillo, "Video Thoracoscopy Expedites the Diagnosis and Treatment of Penetrating Diaphragmatic Injuries,” Surgical Endoscopy, Vol. 15, No. 1, 2001, pp. 28-32. doi:10.1007/s004640002090

[12] D. J. Milfeld, K. L. Mattox and A. C. Beal Jr., "Early Evacuation of Clotted Hemothorax," American Journal of Surgery, Vol. 136, No. 6, 1978, pp. 686-692. doi:10.1016/0002-9610(78)90336-7

[13] J. A. Murray, D. Demetriades, J. A. Asensio, E. E. Cornwell 3rd, G. C. Velmahos, H. Belzberg and T. V. Berne, "Occult Injuries to the Diaphragm: Prospective Evaluation of Laparoscopy in Penetrating Injuries to the Left Chest," Journal of American College of Surgery, Vol. 187, No. 6, 1998, pp. 626-630. doi:10.1016/S1072-7515(98)00246-4

[14] R. K. Freeman, G. Al-Dossari, K. A. Hutcheson, L. Huber, M. E. Jessen, D. M. Meyer, M. A. Wait and J. M. DiMaio, "Indications for Using Video-Assisted Thoracoscopic Surgery to Diagnose Diaphragmatic Injuries after Penetrating Chest Trauma,” Annals of Thoracic Surgery, Vol. 72, No. 2, 2001, pp. 342-347. doi:10.1016/S0003-4975(01)02803-X

[15] C. W. Hsiao, S. C. Lee, C. Tzao, J. C. Chen and Y. L. Cheng, "Minithoracotomy with Simultaneous Video-Assisted Thoracoscopic Surgery vs Video-Assisted Thoracoscopic Surgery for Spontaneous Hemopneumothorax," Thoracic Cardiovascular Surgery, Vol. 51, No. 5, 2003, pp. 288-290. doi:10.1055/s-2003-43077 